\title{
Tsafon
}

Revue d'études juives du Nord

$81 \mid 2021$

Des synagogues à travers les âges Lieux de prières, lieux d'études et autres fonctions

\section{Quelques notes historiques sur la Synagogue antique, la maison d'études et le milieu rabbinique en Judée/Galilée au temps de la Mishna et du Talmud}

\section{Emmanuel Friedheim}

\section{OpenEdition Journals}

Édition électronique

URL : https://journals.openedition.org/tsafon/3579

DOI : 10.4000/tsafon.3579

ISSN : 2609-6420

Éditeur

Association Jean-Marie Delmaire

Édition imprimée

Date de publication : 1 juillet 2021

Pagination : 15-22

ISSN : 1149-6630

Référence électronique

Emmanuel Friedheim, "Quelques notes historiques sur la Synagogue antique, la maison d'études et le milieu rabbinique en Judée/Galilée au temps de la Mishna et du Talmud », Tsafon [En ligne], 81 | 2021, mis en ligne le 01 juillet 2021, consulté le 15 septembre 2021. URL : http://journals.openedition.org/ tsafon/3579; DOI : https://doi.org/10.4000/tsafon.3579 


\title{
Quelques notes historiques sur la Synagogue antique, la maison d'études et le milieu rabbinique
}

\author{
en Judée/Galilée au temps de la Mishna \\ et du Talmud
}

Emmanuel Friedheim*

On cherchera dans cette brève étude à établir l'existence de liens corrélatifs entre la synagogue antique (בית הכנסת) et la maison d'études au sein de la société juive en générale et du milieu rabbinique en particulier. Définir ces institutions est une tâche importante pour bien les replacer dans le contexte historique prévalant en Palestine romaine du II $^{\text {ème }}$ au IV ${ }^{\text {ème }}$ siècle de notre ère. Il est communément admis par la recherche historique que jusqu'à la destruction du second Temple de Jérusalem en l'an 70 de notre ère, la synagogue n'était pas destinée à la prière juive communautaire, mais à la lecture de la Tora et à certaines activités sociales, plaçant ainsi en exergue le rôle public et collectif de la synagogue ${ }^{1}$.

\footnotetext{
* The Israel \& Golda Koschitzky Department of Jewish History and Contemporary Jewry Bar Ilan University.

${ }^{1}$ Voir notamment les travaux précurseurs de S. Krauss, Synagogale Altertümer, Berlin Vienne 1922 ; J. Guttman (ed.), The Synagogue - Studies in Origins, Archaeology and Architecture, New York, 1975 ; E. Schürer, The History of the Jewish People in the Age of Jesus-Christ, II, Revised and Edited by G. Vermes, F. Millar \& M. Black, Edinburgh, 1979, p. 423-454. Pour les sources primaires, voir notamment : Josèphe, Vita Iosephii, 280 (éd. D. Schwartz, Jérusalem, 2007, p. 115). Certains critiques ont toutefois tenté de replacer la liturgie au centre des activités synagogales à une période relativement assez haute, voir notamment : F. G. Hüttenmeister \& G. Reeg, Die antiken Synagogen in Israel, Wiesbaden, 1977; G. Hüttenmeister, "Synagogue and Beth Ha-Midrash and their Relationship », Cathedra for the History of Eretz Israel and its Yishuv, 18 (1981), p. 38 (Héb.).
} 
Pour autant, cela ne signifie pas que la liturgie était absente du cadre synagogal. Toute personne, ayant un quelconque sentiment religieux, faisant acte d'une certaine piété, éprouve le besoin de prier. C'est une constante, notamment dans l'Antiquité, les Juifs ne faisant pas exception à la règle. Tant la Bible que les écrits intertestamentaires, notamment les manuscrits de Qumrân, font état de prières. Il s'agissait néanmoins essentiellement de prières et d'invocations relevant de la sphère privée et intime et non d'un rituel rigide offrant un texte identique pour l'ensemble des fidèles. Le culte public était principalement rendu par les sacrifices, offerts quotidiennement par les prêtres dans le Temple de Jérusalem. Une fois le Temple dévasté, et l'arrêt vraisemblable des sacrifices ${ }^{2}$, le milieu rabbinique en pleine formation, pour (re)constituer au plus vite une élite dirigeante, instaura le rite liturgique de la prière publique, la suppléant de la sorte à l'offrande de sacrifices, tentant ainsi d'imposer, bon gré mal gré, un rituel unique aux passages ordonnés (סידור) qui représente, encore aujourd'hui, le rituel de prières pour le judaïsme orthodoxe.

Ceci dit, S. Safrai a bien montré que lorsque les sources tannaïtiques évoquent la synagogue, l'étude de la Tora y est alors présentée comme une fonction principale, bien avant la prière ${ }^{3}$. Ainsi par exemple, nous lisons dans la Tosefta : "[Dans] les synagogues, on ne se conduit pas avec légèreté... On ne doit ni manger, ni boire dans la synagogue, ni même y dormir... mais on y lit, on y enseigne, on y pratique l'exégèse et on $\mathrm{y}$ prononce des oraisons funèbres $»^{4}$. La liturgie synagogale devenant, quant à elle, davantage présente dans les sources talmudiques des III ${ }^{\text {ème-IV }}{ }^{\text {ème }}$ siècles ${ }^{5}$. D'après cet avis, la fonction liturgique de la synagogue finit par s'imposer majoritairement à l'époque talmudique. D. Urman affirme, $a$ contrario, que les fonctions sociales et éducatives de la synagogue demeurèrent centrales, bien plus importantes que celles de la liturgie,

\footnotetext{
2 Voir toutefois : K. W. Clark, « Worship in the Jerusalem Temple after A. D. 70 », New Testament Studies, 6 (1959-1960), p. 269-280 ; S. C. Mimouni, Le Judaïsme ancien du $V I^{e}$ siècle avant notre ère au III siècle de notre ère : Des prêtres aux rabbins, Paris, 2012, p. 496-498.

${ }^{3}$ S. Safrai, À la fin de l'époque du second Temple et à l'époque de la Mishna-Chapitres sur l'histoire de la société et de la culture, Jérusalem, 1983², p. 145. (Héb.).

4 Tosefta Méguila, 18 (édit. Lieberman, p. 353) ; TJ Méguila, 3, 4 (74a) ; TB Méguila, 28a-b.

${ }^{5}$ TJ Berakhot, 4, 4 (8b) : « R. Tanh'um bar Hanina (IV ème siècle) : Chacun [littéralement : l'homme] doit avoir une place bien à lui pour prier dans la synagogue »; Ibid., 5, 1 (8d) : «R. Pinhas au nom de R. Hosh'aya (fin III ${ }^{\text {ème }}$ siècle) [dit] : Celui qui prie dans une synagogue est comparable à celui qui porte en sacrifice une offrande pure », Et al.
} 
depuis l'époque du second Temple, et ce jusqu'à la fin de l'époque talmudique ${ }^{6}$.

Les sources talmudiques font état de synagogues qui n'étaient pas fréquentées par les Sages, notamment celles d'un autre groupe social, celui des Ameï Ha-aretz, jugé dissident du milieu rabbinique au II ${ }^{\text {ème }}$ siècle de notre ère ${ }^{7}$. Devenant une élite, les rabbins auraient alors décidé de s'éloigner de la synagogue en fondant une institution parallèle, celle de la maison d'études, le Beth Hamidrash ${ }^{8}$. Nous y reviendrons.

Pour autant que l'on sache, nul chercheur ne conteste l'existence de la maison d'études, cependant certains y voient un élément à part entière de la synagogue, voire une pièce de la synagogue, qui aurait été réservée exclusivement à l'étude. Selon cette opinion la maison d'études (Beth Hamidrash) fit intégralement partie du complexe synagogal (Beth Haknesset) ${ }^{9}$. Autrement dit, on aurait érigé des synagogues mais non des maisons d'études avec des bâtiments distincts pour cette institution.

Effectivement, de nombreuses synagogues en date des III ${ }^{\text {ème }}-\mathrm{V}{ }^{\text {ème }}$ siècles ont été découvertes lors de fouilles archéologiques ${ }^{10}$, tandis que les cas avérés de maisons d'études autonomes sont bien plus rares ${ }^{11}$. Urman affirme, cependant, que la maison d'études (Beth Hamidrash) constituait, bel et bien, tant un véritable édifice qu'une institution indépendante de la synagogue.

Il est vrai que plusieurs sources rabbiniques témoignent explicitement ou indirectement de l'existence d'une telle institution en marge du milieu synagogal. Tout d'abord la maison d'études est, comme son nom l'indique, destinée à l'étude et à l'enseignement ${ }^{12}$, ainsi qu'à

\footnotetext{
${ }^{6}$ D. Urman, «The Synagogue and Beth Ha-Midrash - Are They One and the Same? », dans A. Kasher, A. Oppenheimer \& U. Rappaport (eds.), Synagogues in Antiquity, Jerusalem, 1987, p. 58. (héb.) ; Idem, « The House of Assembly [beth ha-knesset] and the House of Study [beth ha-midrash] : Are they One and the Same ? », Journal of Jewish Studies, 44/2 (1993), p. 236-257.

${ }^{7}$ Mishna Avot, 3, 10 ; TB Shabbat, 32a ; D. Jaffé, « Les synagogues des 'amei ha-aretz' : hypothèses pour l'histoire et l'archéologie », Sciences religieuses, 32/1-2 (2003), p. 5982.

${ }^{8}$ Urman, (Supra, n. 6), p. 59-60,

${ }^{9}$ Hüttenmeister, (Supra, n. 1 - II ${ }^{\mathrm{ème}}$ publication), p. 38-44 ; Z. Safrai, The Jewish Community in the Talmudic Period, Jerusalem, 1995, p. 186-189. (Héb.).

${ }^{10} \mathrm{Z}$. Ilan en dénombre 175 (Judée et Galilée confondues), cf. Idem, Ancient Synagogues in Israel, Tel-Aviv, 1991. (héb.).

${ }^{11}$ Safrai, (Supra, n. 9).

${ }^{12}$ Genèse Rabba, 63, 9 (éd. Theodor-Albeck, p. 692-693 ; Ibid., 97 (éd. Theodor-Albeck, p. 1207).
} 
l'énonciation de sermons ${ }^{13}$. La maison d'études fut aussi parfois le théâtre d'opérations à caractère socio-communautaire ${ }^{14}$. Certains Sages talmudiques, notamment Rabbi Ami et Rabbi Assi (III ${ }^{\text {ème }}$ siècle), préféraient prier entre les piliers de la maison d'études plutôt que dans les treize synagogues de Tibériade ${ }^{15}$ ! Une autre tradition nous informe que Rabbi Akha (III-IV ${ }^{\text {ème }}$ siècles) enseigna dans la maison d'études, tandis que

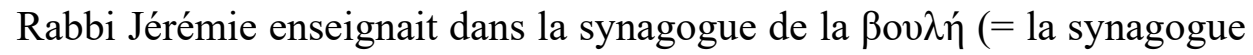
de Tibériade, à proximité du conseil municipal $)^{16}$. On pouvait donc, au temps du Talmud, prier et étudier aussi bien dans la synagogue que dans la maison d'études, deux institutions distinctes l'une de l'autre.

Selon d'anciennes traditions, remontant peut-être à la fin de l'époque du second Temple, il existait déjà une maison d'études sur le mont du Temple avant $70^{17}$. Les textes témoignent également de l'existence de maisons d'études privées de Rabban Yohanan b. Zakkaï à Jérusalem avant la grande révolte (66-73) ${ }^{18}$, de Rabbi Éliézer b. Hyrcanos et de Rabbi Tarfon (II ème siècle) à Lydda (Lod) ${ }^{19}$. Dans la deuxième moitié du II ${ }^{\text {ème }}$ siècle, on apprend l'existence de la maison d'études de Rabbi Méir, probablement à Tibériade ${ }^{20}$. Ensuite, il faut signaler la maison d'études de Rabbi Hiyya (fin II ${ }^{\text {ème }}$ siècle) à Tibériade ${ }^{21}$. De la première génération des Amoraïm du Talmud (III ${ }^{\text {ème }}$ siècle) on évoquera la maison d'études de Rabbi Baniya et de Rabbi Hanina de Sepphoris en Galilée 22 . Lors de la seconde génération des Amoraïm, les sources évoquent la maison d'études à Tibériade de Rabbi Yohanan, décédé en 279 de notre ère ${ }^{23}$. D'autres maisons d'études étaient publiques et ouvertes au plus grand nombre ${ }^{24}$. Ces

\footnotetext{
${ }^{13}$ Mekhilta de R. Ishmael Bo, 16 (éd. Horovitz-Rabin, p. 58-59) ; Tosefta Sota, 7, 9 (éd. Lieberman, p. 193-194) ; TJ Sota, 3, 4 (18d-19a) ; TJ Haguiga, 1, 1 (75d) ; TB Haguiga, 3a).

${ }^{14}$ TB Shabbat, 103a ; Ketouboth, 5a ; Ibid., 63b ; Baba Metzia,28b.

${ }^{15}$ TB Berakhot, 8a ; Ibid., 30b.

${ }^{16}$ TJ Ta'aniot, 1, 2 (64a).

17 Tosefta Sanhédrin, 7, 1 (éd. Zuckermandel, p. 425); Tosefta Haguiga, 2, 9 (éd. Lieberman, p. 383). Voir aussi peut-être dans ce sens: Tosefta Souka, 2, 10 (éd. Lieberman, p. 265); Ibid., 4, 5 (éd. Lieberman, p. 273).

${ }^{18}$ TJ Méguila, 3, 1 (74d).

${ }^{19}$ Cantiques Rabba, 1, 20 (éd. Dunsky, p. 22) ; TJ Érouvine, 4, 4 (22a).

${ }^{20}$ TB Sanhédrin, 11a.

${ }^{21}$ Sifré sur Nombres, 115 (éd. Horovitz, p. 129).

${ }^{22}$ TJ Baba Metzia, 2, 13 (8d) ; TB Ketoubot, $23 \mathrm{a}$.

${ }^{23}$ TB Sota, 22a.

${ }^{24}$ Voir par exemple: Tosefta Passeha, 2 (3) 11 (éd. Lieberman, p. 154) [= maison d'études à Tibériade] ; Tosefta Erouvin, 9, 6 (éd. Lieberman, p. 119) [= maison d'études près de Tiv'on [Beth Shearim en basse Galilée], suivant S. Lieberman, Tosefta Ki-Fshutah
} 
textes semblent tous indiquer que la maison d'études était un édifice indépendant de la synagogue. Il s'agissait parfois, sans doute, d'un édifice monumental, ainsi que les vestiges archéologiques excavés à Dabboura sur le plateau du Golan semblent le montrer. En effet, le linteau de la porte arbore l'inscription suivante: זה בית מדרשו שהלרבי אליעזר הקפר: C'est la maison d'études de Rabbi Éliézer Hakapar] ${ }^{25}$.

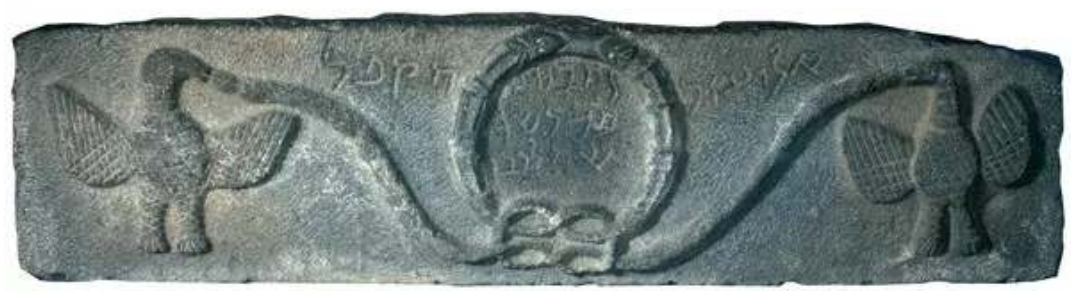

Linteau de la porte avec l'inscription « C'est la maison d'études de Rabbin Éliezer Hakapar» Musée archéologique de Qazrin. Photo d'Emmanuel Friedheim

Ce sage connu de la littérature rabbinique comme œuvrant à l'époque de Rabbi Judah le Prince [180-222 de notre ère env.] est le seul maître dont il est vraisemblablement prouvé par l'archéologie que sa maison d'études a réellement existé ${ }^{26}$. On doit s'interroger sur l'importance relative de cette découverte. En effet, plus de 175 vestiges synagogaux ont été excavés en

- A Comprehensive Commentary on the Tosefta, III, Order Mo'ed, Jerusalem 1992², p. 418. (Héb.) ; TJ Péah, 7, 4 (20b) [= maison d'études à Sepphoris].

${ }^{25}$ Urman, « Jewish Inscriptions from the Village of Dabbura in the Golan », Tarbiz - A Quarterly for Jewish Studies, 40 (1971), p. 406-408. (Héb.) ; Idem, « Jewish Inscriptions from Dabbura in the Golan », Israel Exploration Journal, 22 (1972), p. 16-23 ; D. Meir \& E. Meir, Ancient Synagogues in the Golan Heights, Jerusalem, 2013, p. 110-117. (Héb.).

${ }^{26}$ En dépit du fait que pour certains chercheurs, il ne peut s'agir du maître en question. En effet, Rabbi Éliezer Hakapar est selon les textes rabbiniques un sudiste (דרומאי), c'està-dire de Judée, par conséquent, il est difficile de localiser sa maison d'études sur le plateau du Golan, cf. Z. Safrai, "The Golan in the Roman and Byzantine Period in the Light of Talmudic Sources », dans M. Hartal (ed.), Qaysarin and its Sages : Studies in the Identification of Qazrin and Jewish Settlement in the Golan, Qazrin, 1999, p. 15-16. (Héb.). Z. Safrai est d'avis que cette maison d'études relève plutôt des disciples de Rabbi Éliézer Hakapar, s'agissant ainsi d'une synagogue plus tardive. Sur les difficultés de dater la synagogue en question, cf. Meir \& Meir, (Supra, n. 25), p. 113-114 ; H. Ben David, The Jewish Population in the Golan in the Days of the Mishnah and Talmud in the Light of the Results of Archaeological Surveys in the South of the Golan, Qazrin, 2006, p. 4855. (Héb.). Ben David ne parvient pas à dater avec précision les vestiges de la synagogue de Dabbura. La question reste donc ouverte. 
Palestine romaine ${ }^{27}$, une trentaine dans le Golan ${ }^{28}$, alors que les vestiges des maisons d'étude, scientifiquement attestées par l'archéologie, se comptent, pour ainsi dire, sur les doigts de la main ${ }^{29}$. Selon Urman, l'identification des vestiges synagogaux est souvent erronée en raison de préjugés méthodologiques partant du principe que les vestiges d'édifices juifs de Palestine romaine tardive relèvent presque systématiquement du milieu synagogal. Selon Urman, plusieurs édifices considérés comme synagogaux sont en réalité des maisons d'études et non des synagogues ${ }^{30}$.

La société juive de l'époque de la Mishna et du Talmud était foncièrement plurivoque, avec de nombreux mouvements sociaux et religieux défiant le milieu rabbinique en pleine formation ${ }^{31}$. Certaines synagogues étaient manifestement infréquentables pour les Rabbins, notamment celle de Hammath Tibériade, en date du III ${ }^{\mathrm{ème}}$ siècle de notre ère, avec l'effigie païenne d'Hélios/Sol Invictus au centre de sa mosaïque ${ }^{32}$.

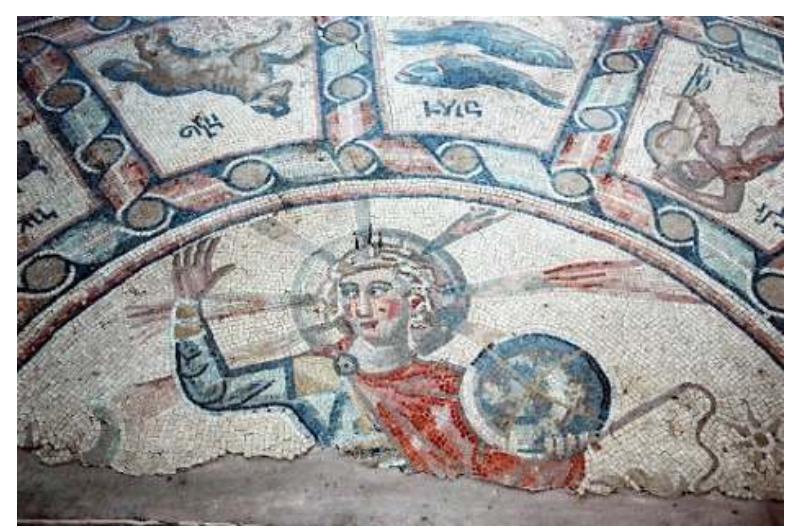

Mosaïque de la Synagogue de Hammat Tibériade (Hammat Tiberias National Park) Photo d'Emmanuel Friedheim

27 Z. Ilan, Ancient Synagogues in Israel, Tel-Aviv, 1991. (Héb.). Et al.

${ }^{28}$ Meir \& Meir, (Supra, n. 25), p. 6. (Héb.).

${ }^{29}$ Safrai, (Supra, n. 9).

${ }^{30}$ Urman, (Supra, n. 6 - I ${ }^{\text {èr }}$ publication), p. 71-75. (Héb.).

${ }^{31}$ Voir en dernier lieu : E. Friedheim, « Le Judéo-polythéisme en Galilée au Temps de la Mishna et du Talmud - Sources complémentaires », Revue des études juives, 179 [3-4] (2020), p. 291-313.

32 E. Friedheim, Rabbinisme et Paganisme en Palestine romaine - Étude historique des Realia talmudiques (I ${ }^{{ }^{r}-I V^{e m e}}$ siècles), Leyde - Boston, 2006, p. 109-159; Idem, « Sol Invictus in the Severus Synagogue at Hammath Tiberias, the Rabbis and Jewish Society : A Different Approach », Review of Rabbinic Judaism, 12/1 (2009), p. 89-128. 
Mais d'autres, notamment celle de Rehob près de Beth-Shéan, était entièrement rabbinique puisque sa mosaïque, exposée aujourd'hui au musée d'Israël, arbore une longue inscription recopiant, pour ainsi dire mot à mot, une halakha rabbinique explicite figurant notamment dans le texte de la Tosefta ${ }^{33}$.

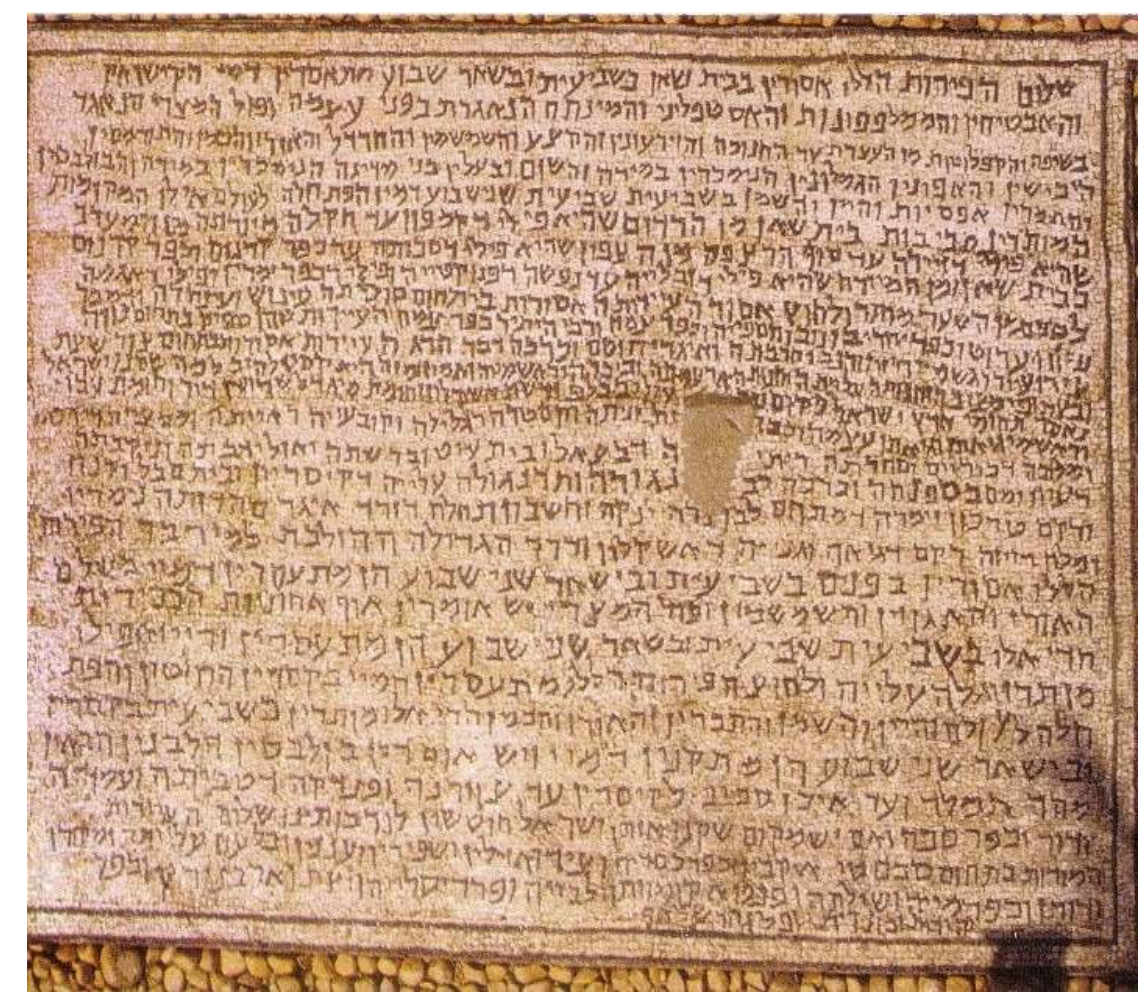

Inscription de la synagogue de Rehob - Musée d'Israël, Jérusalem Photo d'Emmanuel Friedheim

Certains chercheurs soulignent l'importance du judaïsme synagogal en marge du judaïsme rabbinique ${ }^{34}$. Si comme le prétendit D. Urman, la création de la maison d'études rabbiniques (בית המדרש) fut la conséquence

33 Y. Sussman, "A Halakhic Inscription from the Beth-Shean Valley », Tarbiz, 43 (1974), p. 158-188. (Héb.) ; Idem, «The Inscription in the Synagogue at Rehob », Qadmoniot, 8 (1976), p. 123-128. (Héb.) ; H. Misgav « Synagogue Inscriptions from the Mishnah and Talmud Period », dans Y. Eshel et al. (eds.), And Let Them Make Me a Sanctuary - Synagogues to the Ancient Time to the Present Day, Ariel, 2004, p. 49-56. (Héb.).

34 Voir notamment l'étude importante de J. Costa, "Qu'est-ce que le 'judaïsme synagogal' ? », Judaïsme ancien/Ancient Judaism, 3 (2015), p. 63-218. 
de l'impossibilité des Sages à fréquenter les synagogues de groupes déviants de leur milieu ${ }^{35}$, alors devrons-nous considérer davantage la formation du Beth Hamidrash (= Maison d'études) comme une réplique contestataire face à un judaïsme s'exprimant dans des synagogues véhiculant un message divergent de celui du monde rabbinique, tant d'un point de vue doctrinal que rituel.

${ }^{35}$ Notamment les amei-ha-aretz, les judéo-chrétiens et les judéo-polythéistes. 